\title{
MOLECULAR CATASTROPHES AND THE FORMATION OF CIRCUMSTELLAR DUST
}

\author{
Robert E. Stencel \\ Center for Astrophysics and Space Astronomy \\ University of Colorado \\ Campus Box 391 \\ Boulder, CO USA 80309-0391
}

\begin{abstract}
Radiative instabilities due to simple molecules may convert chromospheric material into masering molecules and dust capable of being removed from the star by radiation pressure.
\end{abstract}

Among the four leading explanations for mass loss from red giant and supergiant stars (thermally driven winds, magnetically driven winds, pulsation and radiation pressure on dust grains), there is ample evidence that once dust grains form near such objects, radiation pressure is sufficient to drive them to infinity (Jura 1984). The problem of the formation of such dust is a classic one (Salpeter 1974) which requires understanding the combined roles of radiative transfer, gas dynamics and chemical reactions. Virtually all published studies have only attacked portions of the combined problem.

One key deficiency in previous efforts to understand the formation of circumstellar dust has been in using radiative equilibrium assumptions concerning the nature of the atmosphere underlying the circumstellar envelope (CSE). Jennings and Dyck (1972) asserted that chromospheres are 'quenched' in the presence of dust, based solely on optical Ca II $\mathrm{K}$ line emission. New ultraviolet and microwave analyses (Carpenter et al. 1985; Stencel et al. 1986; Hjellming and Newell 1983; Judge 1986) show the chromospheres of dusty red supergiant stars are persistent, and, unlike the solar chromosphere, may fill the entire volume out toward the base of the CSE (up to several stellar radii). I suggest that this extended chromosphere is prone to instabilities which ultimately result in the formation of dust grains. Such "thermo-chemical" instabilities are analogous to that discussed for the warm and cold phase of the ISM (Glassgold and Langer 1976; Lepp et al. 1985) and in the solar temperature minimum region (Kneer 1983). Kneer described this as " $\mathrm{d} d \mathrm{y}$ namical situation far from radiative equilibrium, caused by molecules and the temperature dependence of their formation."

Compared to ISM conditions, the atmospheres of stars are denser and warmer (log density: 6-12; $\log \mathrm{T}$ : 3-4). Simple molecules like $\mathrm{CO}$, $\mathrm{SiO}, \mathrm{H}_{2} \mathrm{O}$ and $\mathrm{OH}$ are unique in that they have relatively high binding energies, absorb well in the UV and radiate efficiently in 
the IR, and thus act as effective coolants. In high gravity stars like the Sun, the conditions in the upper photosphere tend to associate $C$ and $O$. When this happens, the radiative cooling due to $\mathrm{CO}$ strongly cools the surroundings, leading to the formation of additional $\mathrm{CO}$ molecules which further enhance the cooling until complete $\mathrm{CO}$ saturation is achieved (a runaway process dubbed the "molecular catastrophe" by Kneer 1983 and Muchmore 1986. In the Sun, there is a striking difference between the brightness temperatures in the 2.3 micron $\mathrm{CO}$ band, and atomic features of the upper photosphere. It is this strong temperature sensitivity of molecular opacity which I propose can operate to ultimately lead to the formation of dust at the base of CSE in red supergiant stars.

Joseph Nuth (private communication) has computed the location of thermodynamic equilibrium in the $\mathrm{SiO}=\mathrm{Si}+\frac{1}{2} \mathrm{O}_{2}$ reaction in density and temperature space. By comparing this locus with the temperature density run of model atmospheres for the Sun (VALC, 1981) and a low gravity star, Betelgeuse (Hartmann and Avrett 1984), we discover that the transition line lies just below the solar temperature minimum, but above the corresponding minimum in the low gravity atmosphere. We infer that the process leading to thermal bistability in the solar atmosphere involving $\mathrm{CO}$ is capable of operating with the analogous $\mathrm{SiO}$ molecule in red supergiants like Betelgeuse. Given that $\mathrm{SiO}$ is the basis for the formation of silicate dust grains, this molecular catastrophe may provide the process by which dust is formed and mass loss caused.

VLBI observations of the M4Ie supergiant VX Sgr by Chapman and Cohen (1986) and Lane (1984) are instructive in this context: the SiO masers lie closest to the stellar photosphere (at 1-2 radii), the $\mathrm{OH}$ and $\mathrm{H}_{2} \mathrm{O}$ masers occur farther out (tens and hundreds of radii). Localized $\mathrm{CO}$ catastrophes in the stellar photosphere give rise to pressure perturbations which result in $\mathrm{SiO}$ formation catastrophes in the extended chromosphere of the star. The formation of $\mathrm{SiO}$ in excited states prompts the observed maser emission, and subsequent chemistry anneals the $\mathrm{SiO}$ into clusters and associations like olivine $(\mathrm{Mg}, \mathrm{Fe})_{2} \mathrm{SiO}_{4}$ (Woolf and Nye 1969; Donn and Nuth 1985), which is removed from the star by radiation pressure. The $\mathrm{OH}$ and $\mathrm{H}_{2} \mathrm{O}$ masers result from their formation catastrophes at lower temperatures and densities in the outer chromosphere/CSE where conditions associated with their lower binding energy phase change take place.

The molecular catastrophe description for the conversion of chromospheric gas into molecular masers and circumstellar dust holds promise for a coherent expanation of the formation of these entities and the process of mass loss from cool, high luminosity objects. We will report elsewhere on quantitative simulations of this scenario, in collaboration with David Muchmore and Joseph Nuth, incorporating a full treatment of gas dynamics, radiative transfer and chemical reactions. This work has been supported by CASA at the University of Colorado, for which the author is grateful. I also acknowledge the skillful assistance of Terry Armitage, Susan Barnes, and Michael Van Steenberg in manuscript preparation. 


\section{REFERENCES}

Carpenter, K., Brown, A. and Stencel, R. 1985, Ap.J., 289, 676.

Chapman, J.M. and Cohen, R.J. 1986, M.N.R.A.S., 220, 513.

Clayton, D. 1985 in Interrelationships Among Circumstellar, Interstellar and Interplanetary Dust, NASA Conf. Publ. 2403.

Donn, B. and Nuth, J. 1985, Ap. J., 288, 187.

Glassgold, A. and Langer, W. 1976, Ap. J., 204, 403.

Hartmann, L. and Avrett, E. 1984, Ap. J., 284, 238.

Hjellming, R. and Newell, R. 1983, Ap. J., 263, L85.

Jennings, M. and Dyck, H. 1972, Ap. J., 177, 427.

Judge, P. 1986, M.N.R.A.S., 221, 119.

Jura, M. 1984, Ap. J., 286, 630.

Kneer, F. 1983, Astron. Astrophys., 128, 311.

Lane, A. 1984 in VLBI and Compact Radio Sources, eds. Fanti et al. (Dordrecht; Reidel), p. 329.

Lepp, S., McCray, R., Shull, J.M., Woods, T. and Kallman, T. 1985, Ap. J., 288, 58.

Muchmore, D. 1986, Astron. Astrophys., 155, 172.

Salpeter, E. 1974, Ap. J., 193, 585.

Stencel, R., Carpenter, K. and Hagen, W. 1986, Ap. J., 308 in press.

Vernazza, J., Avrett, E. and Loeser, R. 1981, Ap. J. Suppl., 45, 635.

Woolf, N. and Ney, E. 1969, Ap. J., 155, L183. 